$32^{\text {nd }}$ European Modeling \& Simulation Symposium

$17^{\text {th }}$ International Multidisciplinary Modeling \& Simulation Multiconference

ISSN 2724-0029 ISBN 978-88-85741-44-7 C 2020 The Authors.

DOI: $10.46354 /$ i3m.2020.emss.007

\title{
Railway station capacity assessment utilizing simulation-based techniques and the UIC406 method
}

\author{
Antonín Kavička ${ }^{1, *}$, Roman Divišs ${ }^{1}$ and Petr Veselý ${ }^{1}$ \\ ${ }^{1}$ University of Pardubice, Studentská 95, Pardubice, 532 10, Czech Republic \\ *Corresponding author. Email address: antonin.kavicka@upce.cz
}

\begin{abstract}
The evaluation of rail track layout capacity represents an important part of strategic (long-term) planning or tactical (mediumterm) planning related to railway traffic and railway infrastructure. This contribution pays attention mainly to railway stations that represent crucial transportation points of railway networks. Capacities of railway stations can be assessed (for given traffic scenarios/timetables) according to different qualitative or quantitative viewpoints. Simulation techniques are usually focused on getting total delays of all trains which is closely related to the quality of transport from the passenger perspective. On the other hand, the analytical methods (e.g. based on UIC 406 leaflet) typically calculate occupation rates of selected infrastructure parts, i.e. a certain kind of quantitative assessment is applied. The processed case study deals with capacity detections related to a smaller passenger station. The capacities are investigated with the help of computer simulation and by means of analytical method specified in UIC 406. Both approaches are compared and some recommendations about their application are formulated.
\end{abstract}

Keywords: rail infrastructure capacity assessment; traffic simulation; UIC406 capacity method

\section{Introduction}

The objective of assessing the capacity of railway infrastructure is to evaluate whether the planned traffic intensity and structure can be successfully operated on a given infrastructure.

The simulation technique enables the investigation of a studied traffic, including the occurrence of random disturbances (e.g. train delays) and is able to create data (with respect to the monitored time period) to calculate the total delay of all trains. This operational indicator indirectly reflects the capacity of the system under investigation and enables comparing different traffic scenarios. In this contribution the utilization of the simulation tool MesoRail (Diviš and Kavička, 2015) is described. That tool applies the mesoscopic level of detail.

Analytical methods are rather focused on identifying occupation rates of various parts of the infrastructure, which express a certain reserve for operating higher intensity of traffic flows. Occupation rates can be calculated analytically for example with the application of the compression method. This method is based on the construction of a feasible time compressed timetable derived from the original timetable. Then, for the compressed timetable, the occupation rates belonging to selected infrastructure segments, are calculated analytically. For analytical evaluation of relevant occupation rates the method UIC406 (UIC406, 2013) is exploited and the software tool StaCap406 (Veselý and Bažant, 2015) is used for executing relevant analytical calculations.

The case study examines the capacity of a smaller passenger railway station whereas two selected traffic scenarios (timetables) are considered. Those scenarios are investigated using both approaches: stochastic computer simulation and UIC406 analytical method.

(C) 2020 The Authors. This article is an open access article distributed under the terms and conditions of the Creative Commons Attribution (CC BY-NC-ND) license (https://creativecommons.org/licenses/by-nc-nd/4.0/). 


\subsection{Brief overview of the state-of-the-art}

Many authors deal with the issue of analytical assessment of railway station capacities. That analytical approach is mostly based on the methodology established by UIC - International Union of Railways (UIC406, 2013). On the other hand, there are also methodologies that are created by national railway companies - for example in Germany it is used the Directive 405 (Richtlinie405, 2008). The authors, paying attention to relevant analytical methods, document the use of those methods usually with the help of appropriate case studies. The following publications are focused on analysing analytical methods: (Abril, 2008), (Armstrong and Preston, 2017), (Lindner, 2011), (Malavasi, 2014), (Vesely and Bažant, 2015) and (Heister, 2006).

On the other hand, the utilization of simulation for evaluating capacity properties related to railway stations is documented, for example, by the following publications: (Diviš and Kavička, 2015), (Bažant et al., 2017), (Song, 2014), (Kaakai, 2007), (Adamko, 2008). In this context, various simulation tools, which operate on different levels of detail (mesoscopic or microscopic) are exploited.

\section{Traffic simulation investigating rail infrastructure capacity}

Computer simulation enables stochastic examinations of various traffic scenarios related to a given track infrastructure. Getting relevant traffic features (monitored by the suitable operational indicators) of considered scenarios represents one of the ways of investigating infrastructure capacity. There is presented a utilization of the simulation tool MesoRail for the needs of rail infrastructure capacity explorations within this contribution.

\subsection{Simulation tool MesoRail}

Simulation software tool MesoRail (Diviš and Kavička, 2015) is specialized in mesoscopic simulations reflecting the traffic namely in railway nodes and restricted segments of railway networks.

The traffic conflicts (e.g. platform tracks assignments to delayed arriving trains) can be resolved by applying static Priority Lists $(P L)$ storing alternative resolution variants (e.g. different train routes leading to different admissible platform tracks). The sorting of those priority lists reflects a suitability degree of relevant solutions to be applied (Bažant et al., 2019). The mentioned lists are formed by railway experts within relevant editor. Thus, during simulation experiments dynamic decision-making processes are carried out utilizing predefined priority lists.

On the other hand, Reflective Nested Simulations (RNS) can solve the conflicts as well (Diviš and Kavička, 2016). When a conflict occurs (within so called main simulation) admissible variants of the conflict solution are identified. Then, the batch of nested replications generated for each variant is carried out. Those replications run for a limited time (simulation lookahead). Upon completion, the simulation results are evaluated, and the main simulation continues with the best solution variant (according to a given optimisation criterion).

During simulation experiments the delay increments of all trains are observed. The delay increment of a train is represented by the difference between the delay at the input into and output from the simulating system. Each train is connected with a weight reflecting its importance in the traffic. The weights were selected as follows: $w_{\text {Express }}=1.8, w_{\text {Regio }}=1.0$ and $w_{\text {Cargo }}=0.2$. The weights are specified in the directive SM124-Railway Capacity Assessment that was issued by the state corporation Rail Infrastructure Manager (Správa železnic, S.o.) in the Czech Republic. When the life cycle of a train is over, the resulting delay increment is multiplied by the corresponding weight and the product represents an input into the statistical processing. In order to evaluate the summary results of different traffic scenarios the statistical indicator Average Total Weighted Delay Increment (ATWDI) is utilized. Its value is calculated as the arithmetic mean of the total weighted delay increments related to all replications belonging to a distinct simulation experiment. The $95 \%$ confidence interval is also calculated for ATWDI.

\section{Capacity analysis specified within UIC 406 leaflet}

In view of the different concepts and procedures concerning railway infrastructure capacity and the resulting calculations applied by infrastructure managers, UIC Code 406 - Capacity (briefly UIC406) provides a unique approach to capacity evaluation in the shape of the compression method, which is recommended for those infrastructure managers that use IT support in their evaluations (UIC406, 2013). Unlike the theoretical concepts developed, the compression method is applied to real timetables with practical measurements intended to establish a common understanding of infrastructure capacity. The methodology described in UIC406 supports calculations of rail lines and rail nodes capacities based on the same principles.

The capacity of a rail station infrastructure can be assessed for (i) an individual rail switch zone or for (ii) individual station tracks. The basic capacity index is represented by an occupancy time rate (OccRate) that provides the utilization rate of a certain part of infrastructure. The OccRate-index is calculated as a ratio of the total occupancy time (OccTime) related to a relevant track segment and the total time period (TotalTime) considered.

$$
\text { OccRate }=\frac{\text { OccTime }}{\text { TotalTime }}
$$


Occupancy time for switch zone is calculated as one value representing a minimum time period, within the frame of which it is possible to realize all tasks according to a given timetable.

The capacity (evaluated by OccRate indicator) can be appropriately calculated if a given timetable does not include any conflicts. The timetable defines individual tasks (related to individual trains) and the order of those tasks. The capacity assessment procedure is based on changing time stamps of individual tasks so that they follow each other in the shortest possible time intervals (i.e. a certain kind of a timetable compression is carried out). The first task is considered with its original time stamp and all other tasks' time stamps are changed so that they immediately follow previous tasks (the time headways and technological times are correctly realized). Individual tasks occupy infrastructure elements (tracks, switches) in the switch zones, whereas each switch can serve only to one task at one point in time. An element can be occupied by another task only after its proper release.

After making the timetable compression a total time that is needed to serve all tasks is reduced. During compression the following rules are applied:

- All planned tasks must be carried out.

- During compression the task is moved (as a whole) on the time axis.

- Original order of the tasks which use the same infrastructure elements must be kept.

- Technological times must be kept - an occupancy of element by the next task can start after previous proper releasing.

- Two tasks (that do not use any common element and their parallel execution is not excluded due to any other reason) can be executed in parallel or can be executed in a reverse order.

\subsection{Methodology focused on railway stations}

The approaches specified within UIC406 establish an exemplary methodology to calculate (among other things) occupancy time of switch zones. It is based on the fact that each individual task represents movement of so-called typical train. There is only limited amount of such typical trains and there is also information about critical element - the element that determines minimal duration (interval) when the latter of two trains can start its ride across switch zone after the former train.

It means in practice that it is needed to create for each single typical train the set of time intervals that represent periods when other trains are not able to realize their rides. After determining those intervals, they are considered occupancy times of elements within a given switch zone related to each single train. Certainly, a particular topology of that switch zone has to be reflected. Finally, the matrix of minimum intervals between couples of all consecutive trains is created.

In order to determine relevant occupancy times, the calculation starts with the first planned task (the first train movement). After that the earliest feasible starting time point is computed for each further task.

\subsection{Software tool StaCap 406}

The specialized software tool StaCap406 is utilized (Veselý and Bažant, 2015). That tool is focused namely on computations related to switch zone capacity assessments according to the rules of UIC 406 methodology (UIC406, 2013). The values of OccRateindex can be computed also for station tracks. The tool processes input data and calculate the capacity indicators completely without user interaction. In addition, the graphical representation of the situation before and after compression is depicted within two separate panels (Fig. 4). All results (including the occupation time of each track element after compression) can be exported in tabular form within a corresponding output file.

The form of the visualization of the occupation time windows fully corresponds to examples specified in the UIC 406 leaflet. Vertical axis shows the time. Horizontal axis represents relevant metric distances within the railway station. The occupation time of each track element is shown by coloured rectangles (blocks). Blocks that are related to a single train are linked together. The upper edge of the rectangle represents a start occupation time and the bottom edge represents an end time for that occupation. The horizontal position and size of the rectangle corresponds to the position of the track element in the layout of the station. Different colours are used to distinguish individual trains (Fig. 4) within the relevant scheme.

Input data is stored the following files characterizing each switch zone. Those files are placed in a common directory and are composed of the following data:

- List of trains that are considered in the calculation related to a given switch zone. The order of trains corresponds to a given timetable. The order is determined by occupation time of train routes within the frame of a particular switch zone.

- List of track elements. The track elements are represented by switches, station tracks and line track sections. The calculation is always focused only on the occupancy of switches. Occupancy of the other track elements can be optionally obtained.

- List of the occupation time windows for each track element related to all relevant trains. It contains the following information for each occupancy: train number, track element label, start time and end time of the occupation window.

The compression of the examined timetable and computations of indicator values are performed 
automatically after selecting the directory that contains the relevant input data files. The resulting compression is then visualized.

\section{Case Study}

The case study investigates a smaller prototype railway station, that is inspired by a real passenger station (Zdice) in the Czech Republic. The track infrastructure is depicted in Figure 1, whereas it is interconnected with 3 railway lines (West Line 1, East Line 1, East line 2). In addition, the infrastructure of the studied system involves also further parts (not visualized in Figure 1):

- three simplified border stations (Station West 1, Station East 1, Station East 2) and

- the mentioned rail lines connecting border stations with the prototype station.

The prototype station is composed of two rail switch zones (Switch zone West, Switch zone East) and eight station tracks (numbered as \#1-\#8) that can be exploited for the sojourns of trains. The dead-end track \#7 is accessible only from east rail lines. Other mentioned station tracks are utilized as passable tracks and they are accessible from all line tracks.

The capacity is investigated with regard to two different scenarios/timetables that specify rail traffic within the morning peak hours ( 8 a.m. -10 a.m.) and they are focused on a highly intensive suburban transport. The trains involved in both timetables are of the following categories: regional trains (Regio), express trains (Express) and freight trains (Cargo). The mentioned scenarios have the following essential characteristics.

\section{Scenario 1 (SCO1)}

It is based on the majority of transit regional trains (Fig. 2).

- A total of 46 trains are included (4 Cargo trains, 32 Regio trains, 10 Express trains).

- The utilized station tracks (according to a static track occupation plan): \#1, \#2, \#3.

\section{Scenario 2 ( $\mathrm{ScO} 2)$}

It operates mainly the regional trains which originate or terminate in the studied station (Fig. 3).

- A total of 41 trains are included (4 Cargo trains, 27 Regio trains, 10 Express trains).

- The utilized station tracks (according to a static track occupation plan): \#1, \#2, \#3, \#4, \#5, \#7.

\subsection{Features of simulation experiments}

The implemented simulating system within MesoRail tool reflects the mentioned prototype station, adjacent rail lines and border stations. The declared scenarios are examined within relevant simulation experiments which have the following features:

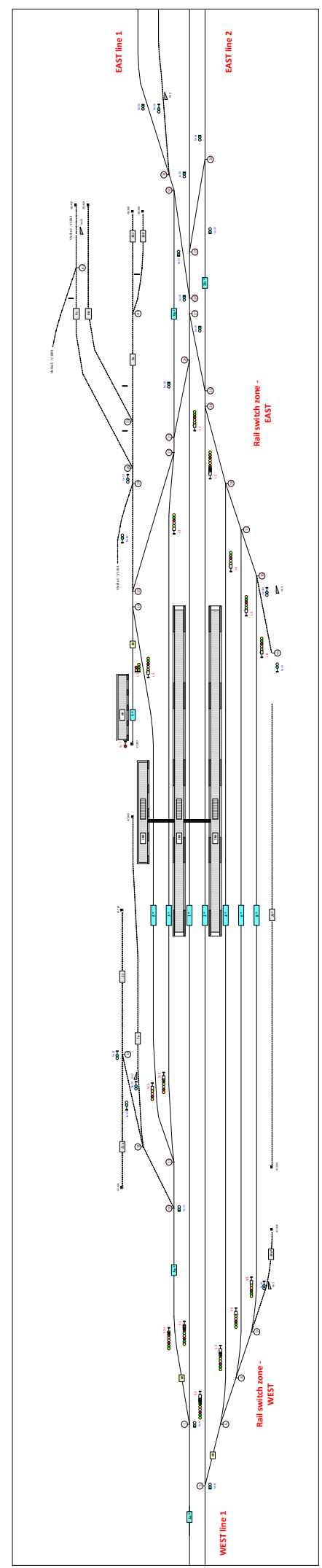

Figure 1. Track layout of the prototype station 
- Each simulation trial (replication) calculates with randomly delayed arriving trains. The utilized train delays match to demands, which are involved in the directive SM124. In this context, each arriving train in the simulating system disposes of two following data items:

- The probability of a delay occurrence associated with the considered train (the relevant decisions are carried out by the generator of pseudorandom numbers conforming to the Bernoulli probability distribution).

- The delay value which is obtained from the generator corresponding to the exponential probability distribution.

- Warming up period of each main simulation run (before collecting data items for relevant statistical analysis) takes 2 hours of simulation time.

- In addition, each scenario has two variants: (i) PL and (ii) RNS. PL variant (Priority Lists) applies priority lists for conflict resolutions (Bažant et al., 2019) and RNS variant (Reflective Nested Simulations) utilizes predictive nested simulations for solving traffic conflicts (Diviš and Kavička, 2016). Nested simulations utilize one level of nesting and each investigated solution variant is examined by the batch of 20 nested replications.

- Within the frame of both scenarios the alternative station tracks, which differ from the static occupation plan (a supplementary material to each timetable), can be assigned to delayed arriving trains (during stochastic simulation experiments). There are the following admissible sets of alternative assignments:

- Sc01: \#1, \#2, \#3, \#4, \#6, \#8.

- Sco2: \#1, \#2, \#3, \#4, \#5, \#6, \#7, \#8.

- Replications of one scenario are always identically parameterized except of the seeds of pseudorandom number generators which differ from each other. There are calculated 100 replications for each scenario/experiment.

\subsection{Procedures applied within analytical calculations}

The elementary data items related to tasks (i.e. train movements) involved in individual scenarios/timetables were obtained from Rail Infrastructure Manager. Those data items describe train rides through individual rail switches, station tracks and rail lines (they are needed for analytical calculations associated with UIC 406 methodology). In addition, occupation times of the mentioned track elements were acquired as well.

The calculations of compression method considered a phased release of train routes.

Some train rides or shunting rides are supposed to be divided into more parts in order to make correct calculations of timetable compressions. It is essential mainly in the cases when the train routes (or shunting routes) are built piecewise within the frame of one switch zone. That situation can emerge if the train (or shunting unit) makes reverse movements.

\subsection{Calculations of capacity indicators}

For the presented traffic scenarios (ScO1 and SCO2), the infrastructure capacity of the railway station was examined using both the computer simulation method and the analytical method UIC406. The calculations of each of these methods were evaluated using different operational indicators:

- The ATWDI indicator (Average Total Weighted Delay Increment), which summarizes the delay increments of all trains, was calculated based on the evaluation of the results of simulation experiments. The values of this indicator can be further decomposed for different train categories (Express, Regio, Cargo) and further recalculated "unweighted" average delay increment for one train (ADI - Average Delay Increment). These averages per train can be referred to as: $\underline{A D I_{\text {Express }}, A D I_{\text {Regio }} \text { and } A D I_{\text {Cargo. }} \text {. The critical limit }}$ values for these sub-indicators for peak periods are specified in (SM124, 2019):

- $A D I_{\text {Express }}=1.5$ minutes

- $A D I_{\text {Regio }}=3$ minutes

- $A D I_{\text {Cargo }}=7.5$ minutes

- The OccRate indicator (Occupancy Time Rate), which focuses on quantifying the occupancy rate of switch zones and station tracks, was calculated using the analytical procedure according to the UIC406 method. This indicator represents a quantitative evaluation of the traffic rate with respect to the examined infrastructure. From its values it can be deduced how the capacity of the monitored track equipment is used. The critical limit value $($ OccRate $=0.60)$ indicates a heavily overloaded device, the optimal limit value $($ OccRate $=0.40)$ expresses the optimal load of the device. The stated limit values are defined in (SM124, 2019).

The elaborated results from the processed calculations (with respect to the examined scenarios of railway traffic - ScO1, ScO2), which are based on the application of computer simulation and analytical method UIC406, are given in Table 1. 


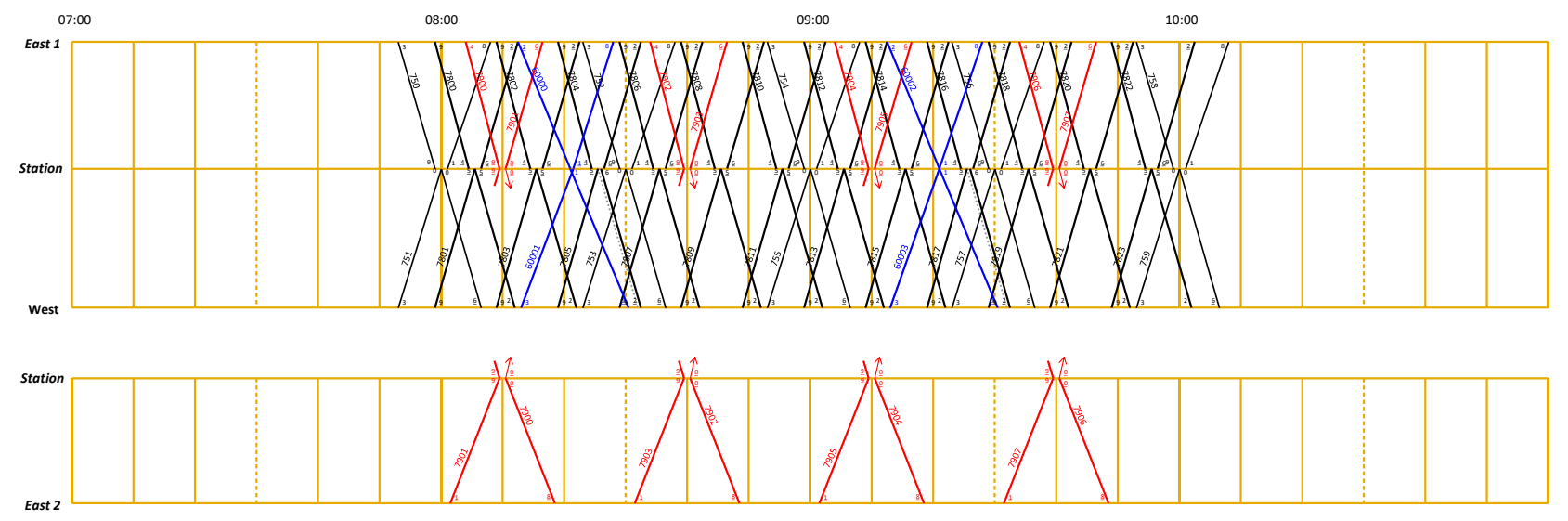

Figure 2. Graphic timetable related to the scenario Sc01
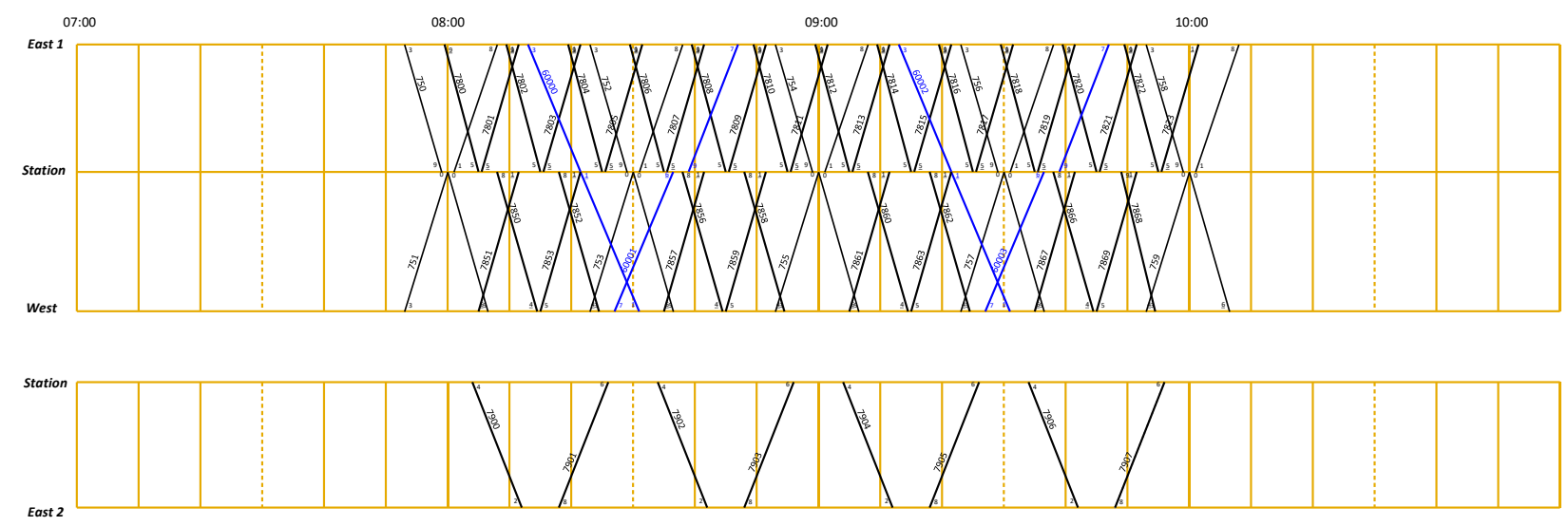

Figure 3. Graphic timetable related to the scenario Sc02

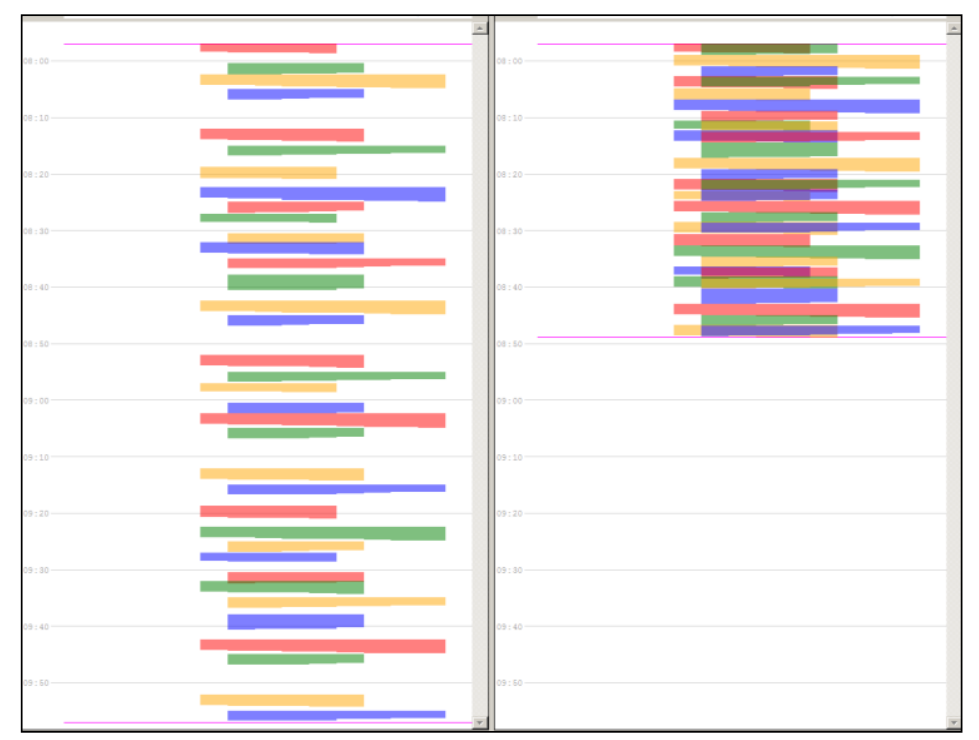

(a)

(b)

Figure 4. Original tasks (a) and compressed tasks (b) within West switch zone (scenario Sc02) 
Table 1. The values of traffic indicators

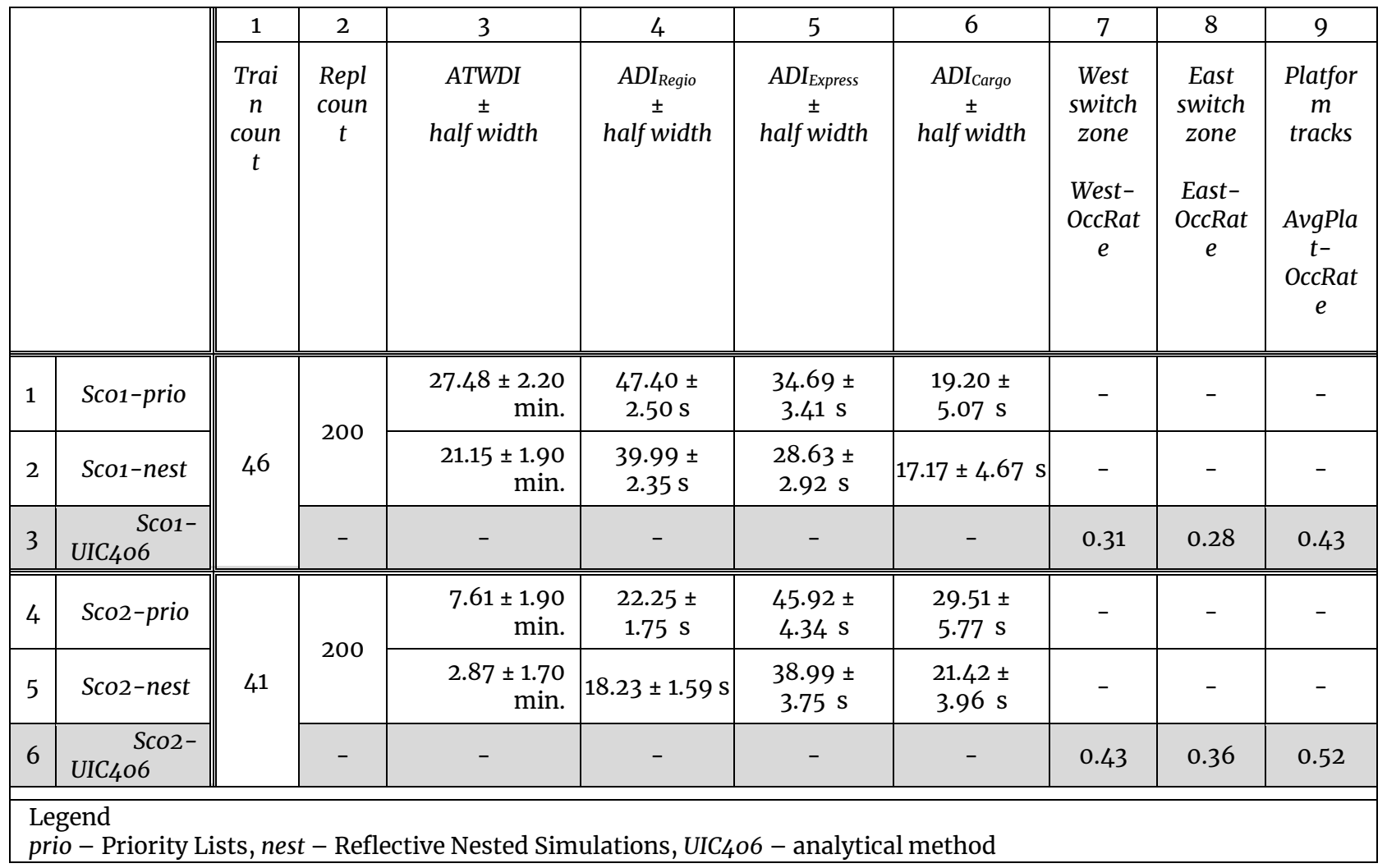

The structure and contents of the presented table can be commented as follows:

(Column 1) The examined count of trains (input parameter).

(Column 2) The applied number of replications within simulation experiments (input parameter).

(Column 3) The computed ATWDI-values regarding relevant scenarios (the corresponding $\quad 95 \%$ confidence interval is stated as well - its half width is quantified).

(Columns 4- The calculated average delay 6) increments per train (for different train kinds).

(Columns 7- The evaluated OccRate-indicators for 9) different parts of the station track layout.

(Rows 1-2) The results of simulations reflecting the scenario Sco1. Simulations apply either (i) static Priority lists for optimisations related to selecting alternative train routes or (ii) the method of recursive/nested simulation for the mentioned kind of optimisations.

(Rows 4-5) The analogical results with regard to the previous cases (Row 1-2) associated with the scenario Sco2.

(Rows 3,6) The results of analytical calculations (applying UIC406 method) linked with scenarios Sc01, Sco2.

\subsection{Interpretation of results}

When inspecting the use of railway station infrastructure capacity in relation to the considered railway traffic scenarios, which often need to be compared with each other, it is typically possible to examine:

- feasibility of the given scenarios on the investigated infrastructure,

- qualitative traffic indicators (associated mainly with the assessment of train delays), which are particularly important in passenger transport and reflect a certain comfort of passengers,

- quantitative operational indicators (evaluating namely the use of infrastructure), which reflect the usage of selected parts of the track layout.

The processed results from the executed calculations (considering the examined scenarios of 
railway operation - Sc01, Sc02) can be evaluated as follows.

\section{Evaluation of the results related to the Scenario 1 (Sco1)}

The operation following this scenario can be assessed as very good, both from the point of view of qualitative indicators (none of the $A D I$-indicators reached the critical limit values) and from the point of view of quantitative indicators. The values of the OccRateindicators are well below the critical limit values, while West-OccRate and East-OccRate did not even reach the optimal limit value (0.40). The value of the AvgPlatOccRate indicator (0.43) is just above the limit of the optimal limit value.

Evaluation of the results related to the Scenario 2 (Sco2) The second scenario enables to implement the relevant railway operation at a very good level. ADI-indicators did not reach the critical limit values. The indicators West-OccRate (0.43) and East-OccRate (0.36) reached values that are in the immediate vicinity of the optimal limit value. In contrast, the AvgPlat-OccRate indicator (reflecting the average utilization rate of platform tracks) reached a value of 0.53 , which is roughly in the middle of the interval between the optimal $(0.40)$ and critical (0.60) limit values.

\section{Mutual comparison of the results linked to scenarios Sco1, ScO2}

The discussed scenarios apply a relatively different traffic organization. While scenario Sco1 predominantly applies (with respect to the investigated railway station) transit regional traffic, scenario $\mathrm{ScO} 2$ involves regional trains which originate or terminate in the mentioned station.

When comparing the traffic variants that correspond to the scenarios ScO1 and ScO2, the following facts can be stated:

(1) The computer simulation method, which examines train delay increments, shows better optimization results (associated with minimization of train delay increments) when applying the nested simulation method than static priority lists.

(2) In view of the ATWDI-indicator, the scenario Sco2 shows significantly better results than Sco1. Thus, in terms of overall passenger comfort, ScO2 represents a better option than Sco1.

(3) From the viewpoint of the utilization rate linked to various parts of infrastructure, the traffic variant associated with the scenario Sco1 has higher capacity reserves.

\section{Conclusions}

In conclusion, it can be stated that for the infrastructure capacity assessment the use of appropriate simulation and analytical methods in combination with each other can be recommended.
Stochastic simulations, which examine primarily the average increments of train delays, can assess the quality of traffic for the relevant scenarios, especially from the passengers' point of view.

Analytical methods for examining infrastructure capacity (represented, for example, by the UIC406 method) are focused on assessing the utilisation rate of important infrastructure components. Such an assessment is crucial for railway infrastructure managers.

Thus, for a complex assessment of the traffic capacity related to the railway infrastructure it is suggested to apply both evaluation approaches and make appropriate decisions based on an integrated assessment of their results. These decisions may be related to the prepared variants of timetables (for medium-term/tactical planning) and to the planned variants of infrastructure reconstructions (for longterm/strategic planning).

\section{Acknowledgments}

The work was supported from the project PosiTrans: ERDF/ESF Cooperation in Applied Research between the University of Pardubice and companies, in the Field of Positioning, Detection and Simulation Technology for Transport Systems (CZ.02.1.01/0.0/0.0/17_049/0008394).

\section{References}

Abril M. et al., 2008. An assessment of railway capacity. Transportation Research Part E: Logistics and Transportation Review. 44, 5 (Sep. 2008), 774-806

Adamko N., Klima V., 2008. Optimisation of railway terminal design and operations using Villon generic simulation model. Transport. 23, 4 (Dec. 2008), 335-340

Armstrong J., Preston J., 2017. Capacity utilisation and performance at railway stations. Journal of Rail Transport Planning \& Management. 7, 3 (Dec. 2017), 187-205

Bažant et al., 2017. Calculation of the capacity of switch area within railway stations with use of simulation methods. In 29th European Modeling and Simulation Symposium, EMSS 2017. Rende: CAL-TEK SRL, 316322.

Bažant M. et al., 2019. Simulation-Based Rail Traffic Optimizations Applying Multicriterial Evaluations of Variants. MENDEL. 25, 1 (Jun. 2019), 139-146.

Diviš R., Kavička A., 2015. Design and development of a mesoscopic simulator specialized in investigating capacities of railway nodes. Proceedings of the European Modeling and Simulation Symposium, 52-57. September 21-23, Bergeggi, Italy.

Diviš R., Kavička A., 2016. The method of nested simulations supporting decision-making process 
within a mesoscopic railway simulator. Proceedings of the European Modeling and Simulation Symposium, 100-106. September 26-28, Larnaca, Cyprus.

Heister G., 2006: Eisenbahnbetriebstechnologie. Bahn Fachverlag, Heidelberg/Mainz.

Kaakai F. et al., 2007. A hybrid Petri nets-based simulation model for evaluating the design of railway transit stations. Simulation Modelling Practice and Theory. 15, 8 (Sep. 2007), 935-969.

Lindner T., 2011. Applicability of the analytical UIC Code 406 compression method for evaluating line and station capacity. Journal of Rail Transport Planning \& Management. 1, 1 (Nov. 2011), 49-57.

Malavasi G. et al., 2014. A synthetic approach to the evaluation of the carrying capacity of complex railway nodes. Journal of Rail Transport Planning \& Management. 4, 1-2 (Aug. 2014), 28-42.

Richtlinie405, 2008. DB Netz AG: Richtlinie 405: Fahrwegkapazität.

Sm124, 2019. Rail Infrastructure Administration (SŽDC): Directive SM124 - Railway Capacity Assessment, Prague, The Czech Republic

Song H. et al., 2014. Carrying capacity of railway station by microscopic simulation method. 17th International IEEE Conference on Intelligent Transportation Systems (ITSC) (Oct. 2014).

UIC406, 2013. UIC Code 406 - Capacity, 2nd edition. International Union of Railways (UIC), Paris, France.

Veselý P., Bažant M., 2015. Switch area capacity assessment using UIC 406 - the procedure and software tool Perner's Contacts, vol. 10, no. 4, 98106. 